\title{
Refinements of sea state statistics for marine renewables: A case study from simultaneous buoy measurements in Portugal
}

\author{
Jean-Baptiste Saulnier ${ }^{\mathbf{a}, *}$, Marc Prevosto ${ }^{\mathbf{b}}$ and Christophe Maisondieu ${ }^{\mathbf{b}}$
}

\author{
${ }^{a}$ Renewable Energy Group, Camborne School of Mines, C.E.M.P.S., University of Exeter, Cornwall Campus, \\ Penryn, Cornwall TR10 9EZ, United Kingdom \\ ${ }^{\mathrm{b}}$ IFREMER, Brest Centre, DCB/ERT/HO, BP 70, 29280 Plouzané, France \\ *: Corresponding author : Jean-Baptiste Saulnier, email address : j.saulnier@exeter.ac.uk
}

\begin{abstract}
:
This paper presents a refined description of the wave climate observed in a Portuguese location for marine energy projects. The data used in this case study were derived from 3-month in situ measurements carried out using three directional buoys that have been deployed simultaneously - yet independently (various buoy types and water depths) - on the Atlantic coast in the neighbourhood of Figueira da Foz. Two ways of reporting the statistics were adopted. In the first one, each recorded sea state was summarized as a set of global parameters accounting for energy, mean frequency and direction, spectral bandwidth and directional spreading related to the main ongoing wave field considered as unimodal. In the second one, each sea state has been decomposed into its own wave components - swells and wind-sea - which are characterised separately and individually by the same set of parameters as previously. Besides the adopted data processing techniques and the illustration of such thorough wave climate description modes, the paper also addresses the advantages and limits of each adopted parameterization in the frame of simultaneous and independent in situ wave measurements.
\end{abstract}

\section{Highlights}

Wave statistics produced considering unimodal and multimodal sea-state descriptions. Spectral bandwidth and directional spreading are included in both descriptions. Spectral bandwidth is significantly correlated to wave energy/peak period. W Wave directional spreading is difficult to estimate in absolute terms. Directional artefacts are obtained with Maximum Entropy Methods in some cases.

Keywords : Sea state; Directional spectrum; Wave systems; Spectrum partitioning; Spectral bandwidth; Directional spreading 


\section{Introduction}

Since the early years of the marine energy exploitation, the need of an accurate description of the wave climate has always been crucial. For wave energy extraction in particular, the knowledge of at least a few sea state parameters like the significant wave height $\mathrm{Hs}$, the peak period $T p$ (or energy period $T e$ ) and, possibly, the peak direction $\theta p$ of the main wave train propagating through the exploitation area, is capital. The collection of such data in time - mostly realized by in situ measurements (e.g. buoys, gauge arrays...) - permits to build up basic statistics on the sea states occurrence rate. Two-entry diagrams are formed, like $\mathrm{Hs}$ $\mathrm{Tp}, \mathrm{Hs}-\mathrm{Te}$ or Hs- $\theta \mathrm{p} . .$. in which each cell gives the expected probability of observing such sea states, see e.g. [1,2]. If a wave energy developer is able to estimate the mean performance of his device in each so-defined sea state, he is then able to predict the long-term amount of converted wave energy available to the electrical grid. This figure is supposed to give credit or not to the whole exploitation project.

Now, a sea state cannot systematically be synthesized by a simple two- or threeparameter description. This is firstly due to the fact that any body oscillating in water - and therefore, any marine energy structure - may behave very differently in two sea states of same $\mathrm{Hs}$ and Tp (or Te), out of directional considerations, see [3]. Additional characteristics have to be included into the sea state characterisation indeed, so that the mean response and performance of the converter may be assessed quite accurately. A floating structure, resonant by nature, is subject to the wave excitation within a certain frequency (or period) band. The fact the ongoing waves correspond to either a broad- or narrow-banded process may dramatically change the mean dynamical behaviour of the structure. In more concise terms, for same wave energy level (Hs), peak or energy period (Tp, $T e$ ) and possibly same peak or mean wave direction $(\theta p, \theta \mathrm{m})$, the spectral bandwidth of the whole wave field inevitably appears as a relevant extra-parameter for characterising a sea state, especially when the body is little sensitive to wave directionality and the sea state is dominated by one wave system (nearly unimodal seas). Likewise, when the devices are significantly sensitive to the directionality, the directional spreading of the wave components is to be taken into account in the sea state characterisation. Accordingly, an extensive description of the whole sea state requires these two last properties, in addition to the three ones previously introduced.

The second reason why the classical description is incomplete is the fact a sea state often is made of the superimposition of coexisting wave systems, as independent swells and a possible local wind-sea. Thus, if a windy sea state is made up by the conjunction of a windsea and a swell, - both propagating along far different directions but overlapping in frequency, - a floating structure may be influenced by one of them more than the other one. Typically, wave energy prototypes being currently tested at sea operate in 5 to 10 s waves, which means that they preferentially respond to wind waves, and to a lesser extent, to ends of long-travelling swell trains. In such cases, overall information about the sea state is only partly relevant: each wave system has to be identified and characterised properly so that its influence upon the device may be better identified and emphasized.

Both of these descriptions - referred here as overall (or unimodal) and multi-system - are adopted in this work, which involves wave data obtained by in situ buoy measurements 
(displacement Waverider and heave-pitch-roll Wavec) near the Portuguese location of Figueira da Foz at various water depths (20 to $\sim 90 \mathrm{~m})$. From spectral analysis coupled to directional re-composition, the directional wave spectra $S(f, \theta)$ can be estimated and processed to produce: 1 / an overall extended characterisation of the sea state, and 2 / a wave system characterisation - swells and wind-seas - thanks to multi-system analysis. Both result in new statistics as new 2-entry diagrams mostly, which are presented in the following. This case study allows for a deeper analysis of the local wave climate (here, a short one indeed, three months only) but also addresses the advantages and limits of the adopted parameterisations. Also, the difficulties related to dealing with independent and separate data sources as different buoy types and water depths are emphasized.

\section{Wave data processing}

\subsection{Sea state and wave spectrum}

A sea state is defined as stationary and homogeneous wave conditions (sea surface elevation $\eta$ ) over a limited time duration and geographical area. It is commonly admitted that a sea state meets these properties during 1 to 3 hours and over some tens of square kilometres, out of extreme conditions like rough storms etc. where it may vary much faster. Thus, any in situ measurement of the wave field - using (non-)directional buoys, pressure sensors, gauge arrays... - carried out over a few tens of minutes is generally assumed to characterise the seastate reasonably well up to the next one or two hours. Of course, the more frequent the measurements, the finer the analysis.

In linear wave theory, the wave spectrum $S(f, \theta)$ (also called spectro-directional energy density, expressed in $\left.\mathrm{m}^{2} \cdot \mathrm{Hz}^{-1} \cdot \mathrm{rad}^{-1}\right)$ is used to describe the distribution of wave energy in the 
frequency-direction plane. Integrating the wave spectrum over frequencies and directions yields the variance of the recorded elevation process

$$
\iint S(f, \theta) d f d \theta=m_{0}
$$

which is confounded with the $0^{\text {th }}$-order spectral moment. Indeed, the spectral moments of $n^{\text {th }}$ order are computed as

$$
m_{n}=\iint f^{n} S(f, \theta) d f d \theta
$$

which are used to calculate some overall wave parameters, as shown in Section 3.

\subsection{Directional wave spectrum estimation}

From the collection of measured field wave data (directional ones here since data from heave-pitch-roll and displacement buoys are used), successive wave spectra in time - that is, a sea state evolution - can be estimated. To facilitate the practical estimation of $S(f, \theta)$ it is common to decompose the spectrum into

$$
S(f, \theta)=S(f) \cdot D(f, \theta)
$$

where $S(f)$ represents the omnidirectional energy spectral density $\left(\right.$ in $\left.\mathrm{m}^{2} . \mathrm{Hz}^{-1}\right)$ - obtained by integrating $S(f, \theta)$ over directions - and $D(f, \theta)$ denotes the directional distribution function (in 
$\left.\operatorname{rad}^{-1}\right)$, which is a function of frequency and direction and satisfies for any frequency $f$ the following condition of normalisation

$$
\int_{0}^{2 \pi} D(f, \theta) d \theta=1
$$

The numerical determination of this function is certainly the trickiest part of the computation of the directional wave spectrum. Indeed, estimating the density $S(f)$ is somewhat easy using (Fast) Fourier Transform and not subject to much uncertainty as soon as the records are sufficiently long. Now, the practical estimation of $D(f, \theta)$ requires a little more care, in particular when resorting to single-point measurement devices, like directional buoys for instance. The directional distribution function, positive and $2 \pi$-periodic, may be decomposed into the Fourier series

$D(f, \theta)=\frac{a_{0}(f)}{2 \pi}+\frac{1}{\pi} \cdot \sum_{j=1}^{\infty}\left[a_{j}(f) \cos (j \theta)+b_{j}(f) \sin (j \theta)\right]$

where the Fourier coefficients are computed as

$a_{j}(f)=\int_{0}^{2 \pi} D(f, \theta) \cos (j \theta) d \theta$

and

$b_{j}(f)=\int_{0}^{2 \pi} D(f, \theta) \sin (j \theta) d \theta$ 
The absolute knowledge of this function therefore implies that of the Fourier coefficients up to a certain order, at least sufficiently high such that their modulus becomes negligible in comparison to the first order. In practice, the determination of high-order coefficients is not simple and requires a complex measuring apparatus, see e.g. [4,5]. Single-point devices such as heave-pitch-roll directional buoys are more frequently used, however. It is shown [6] that such devices permit to estimate - out of the variance spectral density $S(f)$ - the Fourier coefficients of the only $1^{\text {st }}$ and $2^{\text {nd }}$ order: $a_{1}(f), b_{1}(f), a_{2}(f)$ and $b_{2}(f)$. This requires a crossspectral analysis of the buoy's three degrees of freedom, that is, heave, pitch and roll, assumed to stand for the elevation and slopes of the local free surface against the South-North and West-East axes respectively. The buoy therefore records (over $T$ seconds) the data $\left\{X_{1}(t)\right.$, $\left.X_{2}(t), X_{3}(t)\right\}=\{z(t) \equiv \eta(t), d \eta / d y(t), d \eta / d x(t)\}$ where the $(x O y)$ coordinate system describes the plane defined by the ocean's free surface at rest with $x$ and $y$ axes pointing towards East and North respectively, and heave motion $z(t)$ is assumed to stand for the water excursion $\eta(t)$ at point $O$ along the axis normal to $(x O y)$. As an alternative, the buoy may measure its own motions in that plane (surge and sway), so that the cross-spectral analysis is applied to the displacement data $\{z(t) \equiv \eta(t), y(t), x(t)\}$. The proper equations related to a particular set of measured data may be found in [7], in which an exhaustive review of re-composition methods of the directional spectrum $S(f, \theta)$ from in situ spectral estimations is made. Some of these methods are presented here below for the purpose of this study. Their application is not exclusively limited to heave-pitch-roll or displacement buoy data but to any measuring device allowing for the computation of at least the two first Fourier coefficients $\left(a_{1}(f), b_{1}(f)\right)$ of the decomposition of the directional distribution function $D(f, \theta)$.

\subsubsection{Re-composition by truncated Fourier series}


The most immediate re-composition technique consists in estimating $D(f, \theta)$ from equation (5) up to the $1^{\text {st }}$ or $2^{\text {nd }}$ order, according to the available data. Thus, the function $D(f, \theta)$ may roughly estimated as

$D_{t f s}(f, \theta)=\frac{1}{2 \pi}+\frac{1}{\pi} \cdot\left[a_{1}(f) \cos (\theta)+b_{1}(f) \sin (\theta)+a_{2}(f) \cos (2 \theta)+b_{2}(f) \sin (2 \theta)\right]$

This method is generally discarded because the resulting directional distribution often exhibits negative values as well as non-physical secondary peaks. With such a method indeed, it is crucial to know Fourier coefficients of higher order.

\subsubsection{Re-composition by parametric method}

The precise knowledge of the directional spectral shape is tough to derive since some information is missing when measuring the directional properties of the wave field experimentally. Thus, the "true" shape has to be approached as best as possible from a limited amount of field information. A simple way of estimating $D(f, \theta)$ without need of orders higher than one is by fitting a parametric function for each frequency bin. Obviously, such a function imposes a particular shape to the distribution. In addition, sea states made of two coexisting wave systems at close frequencies but distinct directions will not be properly reproduced after re-composition for the spectrum will exhibit one peak only, to which the whole energy will be attributed. However, at particular locations where the sea states do not significantly vary in direction, such a method can be easily implemented to obtain a relevant first guess of the spectral shape.

One of the most popular parametric functions is the $\cos ^{2 \mathrm{~s}}$ function [4], defined as 
$D_{c 2 s}(f, \theta)=\frac{2^{2 s(f)-1} \Gamma^{2}(s(f)+1)}{\pi \Gamma(2 s(f)+1)} \cdot \cos ^{2 s(f)}\left(\frac{\theta-\theta_{0}(f)}{2}\right)$

where $\Gamma$ denotes the Gamma function and $s(f)$ the spreading coefficient, which can be abusively estimated from the $1^{\text {st }}$-order Fourier coefficients as

$s(f) \equiv \frac{r_{1}(f)}{1-r_{1}(f)}$

where $r_{1}(f)=\left|c_{1}(f)\right|$ with $c_{1}(f)=a_{1}(f)+i b_{1}(f)$, and $\theta_{0}(f)$ by

$\theta_{0}(f) \equiv \arg \left[c_{1}(f)\right]$

Similarly, expressions for both parameters can be derived from the $2^{\text {nd }}$ order Fourier coefficients. Figure 1 illustrates this function for various values of spreading $s$ around mean direction zero.

Fig. 1

Alternatively, Gaussian, Poisson, von Mises... and even bimodal distributions may be used instead of the $\cos ^{2 \mathrm{~s}}$ formulation, see e.g. [8]. In the following, the classical $\cos ^{2 \mathrm{~s}}$ function is retained for exemplification.

\subsubsection{Re-composition by Maximum Entropy Methods}


The estimated Fourier coefficients $a_{1}(f), b_{1}(f), a_{2}(f)$ and $b_{2}(f)$ being given for each frequency bin, an infinity of directional shapes may match this set of values. To find out a likely compromise, some authors have applied statistical methods based on the concept of entropy, which may be defined as "a measure of uncertainty for a stochastic variable" (H. E. Krogstad, pers. comm.). Two integral formulations of the entropy function were proposed, the one by J. P. Burg [9] and the second by C. E. Shannon [10,11]. In both cases, the aim is to minimize - or maximize, depending on the adopted definition - the functional under some constraints on $a_{1}(f) \ldots b_{2}(f)$. As discussed in $[7,12,13]$, Burg's formulation - although a straightforward analytical solution of the problem may be derived, see below - leads to very narrow peaks and sometimes produces artificial double-peaks in unimodal sea states. However, it provides a very good resolution for close directional peaks [13]. Shannon's formulation is a bit more complex in terms of numerical resolution, and requires a robust iterative algorithm. The related entropy function is expressed as the integral

$H_{S M E M}(D, f)=-\int_{0}^{2 \pi} D(f, \theta) \ln (D(f, \theta)) d \theta$

while Burg's formulation is given by

$$
H_{B M E M}(D, f)=\int_{0}^{2 \pi} \ln (D(f, \theta)) d \theta
$$

In practice, Shannon's definition (also known as Maximum Entropy Principle in literature) avoids the creation of artificial double-peaked systems and therefore is more reliable for the automatic processing of large field data [7,11]. Let us stress that both Burg's and Shannon's 
entropy formulations yield directional shapes that rigorously match the four first Fourier coefficients, with distinct resulting shapes though. It is shown, indeed, that the solution of the minimization problem takes the form

$$
D_{B M E M}(f, \theta)=\frac{1}{2 \pi} \cdot \frac{\sigma_{e}^{2}(f)}{\left|1-\varphi_{1}(f) e^{-i \theta}-\varphi_{2}(f) e^{-2 i \theta}\right|^{2}}
$$

for Burg's formulation - where $\sigma_{\mathrm{e}}(f), \varphi_{1}(f)$ and $\varphi_{2}(f)$ are frequency-dependent parameters -, and

$D_{S M E M}(f, \theta)=\exp \left[\lambda_{0}(f)+\lambda_{1}(f) \cos (\theta)+\lambda_{2}(f) \sin (\theta)+\lambda_{3}(f) \cos (2 \theta)+\lambda_{4}(f) \sin (2 \theta)\right]$

for Shannon's formulation - where $\lambda_{0} \ldots \lambda_{4}$ are (frequency-dependent) Lagrange multipliers. Both solutions therefore are mathematically correct but not necessarily similar.

\section{Sea state characterisation}

\subsection{Unimodal description: overall characterisation}

In offshore engineering applied to marine renewable energy, it is common to characterise the sea states through a certain number of wave parameters such as the spectral significant wave height $H_{\mathrm{m} 0}\left(\equiv H_{\mathrm{s}}\right)$, defined as

$$
H_{m 0}=4 \sqrt{m_{0}}
$$


the peak period $T_{\mathrm{p}}$ and/or mean energy period here denoted by $T_{-10}\left(\equiv T_{\mathrm{e}}\right)$, calculated as

$$
T_{-10}=\frac{m_{-1}}{m_{0}}
$$

the peak direction $\theta_{\mathrm{p}}$ and/or mean direction $\theta_{\mathrm{m}}$, calculated as

$$
\theta_{m}=\arg \left[\iint S(f, \theta) e^{i \theta} d f d \theta\right] \equiv \tan ^{-1}\left(\frac{\iint S(f, \theta) \sin (\theta) d f d \theta}{\iint S(f, \theta) \cos (\theta) d f d \theta}\right)
$$

and, possibly, the omnidirectional wave power $P_{\mathrm{w}}\left(\right.$ in $\left.\mathrm{kW} \cdot \mathrm{m}^{-1}\right)$, expressed as

$$
P_{w}=\rho g \int c_{g}(f, h) S(f) d f=\rho g \iint c_{g}(f, h) S(f, \theta) d f d \theta
$$

where $c_{\mathrm{g}}(f, h)$ denotes the group celerity of wave frequency $f$, which depends on the local water depth $h(\mathrm{~m})$. Constants $\rho$ and $g$ respectively stand for the sea water mass density $\left(\rho \sim 1025 \mathrm{~kg} \cdot \mathrm{m}^{-3}\right)$ and the gravitational constant. It may be shown that the group celerity in deep water $(h \rightarrow \infty)$ is given by $c_{\mathrm{g}}(f, \infty)=g /(4 \pi f)$. Hence, the approached expression for omnidirectional wave power (in $\mathrm{kW} \cdot \mathrm{m}^{-1}$ ) in deep water

$$
P_{w}=\frac{\rho g^{2}}{64 \pi} H_{m 0}^{2} T_{-10} \approx 0.49 H_{m 0}^{2} T_{-10}
$$

However, it has been shown [3] that, as well as the spectral shape of sea states may vary for a same set of wave parameters such as \{height, period, direction $\}$, the behaviour - 
and therefore, the performance - of a wave energy converter may vary a lot. Thus, the overall characterisation of sea states must also take into account the overall spectral shape in both frequency (spectral bandwidth) and direction (directional spreading). To this end, various bandwidth parameters have been reviewed by the author, among which the equivalent bandwidth $\Lambda$ (in Hz), given by Blackman and Tukey [14], which has been retained in this study. It is computed from the frequency spectrum $S(f)$ as

$$
\Lambda=\frac{m_{0}^{2}}{\int S^{2}(f) d f}=\frac{\left(\int S(f) d f\right)^{2}}{\int S^{2}(f) d f}
$$

To describe the overall spreading of the directional spectrum, the parameter $\sigma_{\mathrm{m}}$ is used here as the mean spreading (in deg) computed as

$$
\sigma_{m}=\arg \left[\int S(f) e^{i \sigma_{1}(f)} d f\right] \equiv \tan ^{-1}\left(\frac{\int S(f) \sin \left(\sigma_{1}(f)\right) d f}{\int S(f) \cos \left(\sigma_{1}(f)\right) d f}\right)
$$

where the $1^{\text {st }}$-order spreading coefficient $\sigma_{1}(f)$ is related to the directional spectrum $S(f, \theta)$ by

$$
\left.\sigma_{1}(f)=\sqrt{2\left[1-r_{1}(f)\right]}=\sqrt{2\left[1-\sqrt{a_{1}^{2}(f)+b_{1}^{2}(f)}\right.}\right]
$$

using equations (3), (6) and (7). The (unimodal) sea state vector

$$
\boldsymbol{\Sigma}_{\mathbf{u}}=\left\{H_{m 0}, T_{-10}\left(T_{p}\right), P_{w}, \theta_{m}\left(\theta_{p}\right), \Lambda, \sigma_{m}\right\}
$$

is therefore constituted. 


\subsection{Multimodal description: multi-system characterisation}

The multimodal description of sea states consists in characterising each wave system individually similarly to the overall description for the whole spectrum considered as unimodal. Assuming a sea state may be decomposed into the linear superposition of independent wave systems, this procedure provides a very fine description of the mean wave field for it takes the underlying multi-system nature of sea states into consideration. Accordingly, a specific analysis for the partitioning of spectra $S(f, \theta)$ into their main components (swells and wind-sea) is required. It is assumed that a spectral peak of energy in the frequency-direction plane stands for a concentration of energy belonging to a particular wave system, each spectral partition being obviously considered as unimodal. Here, a specific application dedicated to Spectral Partitioning for Operational Parameters Identification $\left(\mathrm{SPOP}^{1},[15]\right)$ is used to process histories of (directional) spectral data automatically. As a result, the wave contents in each sea state are summarized into sets of wave parameters similar to overall ones (Eq. (24)), which therefore account for the energy, mean/ period and direction, wave power, spectral bandwidth and directional spreading of each wave system. Then, the whole sea state may be reconstructed by simple superposition of the latter. The last step consists in classifying the systems into either „,swell' or ,wind-sea'.

In SPOP, the partitioning algorithm is based on the steepest ascent path method (or catchment area technique, see [16,17]) applied to the spectral matrix $\left(S_{\mathrm{ij}}=S\left(f_{\mathrm{i}}, \theta_{\mathrm{j}}\right)\right)$ using the watershed routine of the Matlab ${ }^{\circledR}$ Image Processing Toolbox. When the identified partitions are too close from each other (spectral artefacts) or too weak in terms of energy, they are grouped together (see Fig. 2). This yields raw values for energy $\left(H_{\mathrm{m} 0}\right)$, peak period $\left(T_{\mathrm{p}}\right)$ and 
direction $\left(\theta_{\mathrm{p}}\right)$ for each partition. Then, each partition is fitted with analytical shapes against frequency and direction. The frequency fit is based on modified JONSWAP spectra (also called " $\Gamma$-spectra", see [15]) defined as

$S(f)=\frac{\alpha g^{2}}{(2 \pi)^{4}}\left(\frac{f}{f_{p}}\right)^{-5-p} \cdot \exp \left[-\frac{5}{4}\left(\frac{f}{f_{p}}\right)^{-4-p}\right] \cdot \gamma^{\exp \left[\frac{\left(f-f_{p}\right)^{2}}{2 \delta^{2}}\right]}$

with

$\delta=0.07, \quad f<f_{p}$

$\delta=0.09, \quad f \geq f_{p}$

$\alpha \mid \int S(f) d f=\frac{H_{m 0}}{16}$

where $\gamma$ denotes the JONSWAP peak enhancement factor (the higher $\gamma$, the sharper the peak) and $p$ the additional (user-defined or adjusted) decrease factor. The peak period $f_{\mathrm{p}}\left(=1 / T_{\mathrm{p}}\right)$ may be slightly corrected in order to obtain the best fit of the partition spectrum. The energy level $\left(m_{0}\right)$ may also be corrected afterwards according to possible interactions due to energy spectral overlapping after superposition of the wave system spectra, so that the resulting synthetic sea state spectrum is found as close as possible to the original one (see Fig. 3 against frequency).

The low- and high cut-off frequencies (10dB-attenuation at both sides of the peak) $f_{\mathrm{cl}}$ and $f_{\text {ch }}$ of the fitted frequency spectrum are computed and used to form the bandwidth $(\mathrm{Hz})$

$\lambda=f_{c h}-f_{c l}$

\footnotetext{
${ }^{1}$ SPOP was jointly developped by IFREMER and Actimar (http://www.actimar.fr)
} 
A rough estimate of wave power - regardless of the local water depth - is derived here from equation (20) in deep water, as

$P_{w a}=0.42 H_{m 0}^{2} T_{p}$

assuming a standard Bretschneider spectral shape, for which: $T_{-10} \sim 0.86^{*} T_{\mathrm{p}}$.

The directional fit is realised using the $\cos ^{2 s}$ directional distribution function (alternative expression of Eq. (9))

$D(f, \theta)=\frac{\Gamma(s+1)}{2 \sqrt{\pi} \Gamma(s+1 / 2)} \cdot \cos ^{2 s}\left(\frac{\theta-\theta_{p}}{2}\right)$

where the peak direction $\theta_{\mathrm{p}}$ may be slightly corrected in order to obtain the best fit of the directional function. The spreading value (in deg) associated with the fitted function - through parameter $s-$ is also calculated and denoted by $\sigma$.

In the end, each identified partition $i$ is characterised by the vector

$\boldsymbol{\Sigma}_{\mathbf{m}, \mathbf{i}}=\left\{H_{m 0, i}, T_{p, i}, P_{w a, i}, \theta_{p, i}, \lambda_{i}, \sigma_{i}\right\}^{t}$

so that the whole sea state may be represented as the (multimodal) matrix

$$
\Sigma_{m}=\left\{\Sigma_{m, 1}, \ldots, \Sigma_{m, n}\right\}
$$

where $n$ denotes the total number of wave systems making up the sea state. To identify swells and wind-seas, SPOP first segregates both kinds of systems using wind-based criteria - 
separation frequency and angle - under the assumption that the celerity of wind-sea waves cannot exceed the wind's one while propagating along a close direction. This first identification is confirmed in a second time by realizing a wave system tracking based on the time evolution of parameters $f_{\mathrm{p}}$ and $\theta_{\mathrm{p}}$ and by checking the persistence of these (met) criteria in time. Wind-seas are characterised by high-frequency waves, whose wavelength increases i.e. frequency decreases - as time passes by according to the local wind regime $\left(f_{\mathrm{p}}>0.15 \mathrm{~Hz}\right.$ mostly). On the contrary, swells generally correspond to low-frequency wave trains $\left(f_{\mathrm{p}}<0.15 \mathrm{~Hz}\right.$ mostly): according to wave physics, the wave frequency observed at some point is expected to increase for high-frequency waves travel slower than low-frequency ones. Through the observation of the time evolution of peak frequency $f_{\mathrm{p}}$ - and provided the time evolution of peak direction $\theta_{\mathrm{p}}$ is consistent with wind data -, SPOP is therefore able to classify the identified wave systems into wind-seas or swells in any sea state.

Fig. 2

Fig. 3

\section{Data sources and numerical processing}

\subsection{In situ buoy wave data}

\subsubsection{OCEANOR data}

For the purposes of the WAVEMOD project in the 1990s, the Norwegian company OCEANOR deployed two directional Waverider buoys (denoted here by „DW1' and „DW2') at two water depths $(\sim 72 \mathrm{~m}$ and $\sim 20 \mathrm{~m})$ near Figueira da Foz as depicted in Figure 4 . The buoys were operating simultaneously over the period March-May 1994 and recorded heave, 
surge and sway time-series every $3 \mathrm{~h}$ over a duration of $1600 \mathrm{~s}(26 \mathrm{~min}$ and $40 \mathrm{~s})$ at $3.84 \mathrm{~Hz}$ sampling rate. The estimates of $S(f), a_{1}(f), b_{1}(f), a_{2}(f)$, and $b_{2}(f)$ were computed onboard the buoys by block spectral analysis (periodogram technique with edge tapering). The details related to the buoys' deployment and measurements are summarized in Table 1.

\subsubsection{PHI data}

At the same moment - yet independently from OCEANOR -, the Portuguese Hydrographic Institute (Instituto Hidrográfico, ,PHI’) deployed two directional Wavec buoys at two different locations. The first one („DW3') was measuring offshore waves near the OCEANOR buoy DW1 (at 92m depth, see Fig. 4), whereas the second one (,DW4') was placed more in the North, close to the city of Oporto (Matosinhos, Leixões harbour). DW4 stopped measuring at the end of March 1994 while DW3 operated from early March to the end of June 1994. The results related to buoy DW4 are not included in this study however.

The raw record data of these buoys were provided by the PHI. They consist in 1200s time-series of heave $z$, pitch $\varphi$ and roll $\theta$ recorded at $1.28 \mathrm{~Hz}$ sampling rate every $3 \mathrm{~h}$ ( + extra recordings every $30 \mathrm{~min}$ during storms, when $H_{\mathrm{m} 0}>5 \mathrm{~m}$ ). Missing data points in the time series are estimated thanks to cubic-spline interpolation. Then, a block spectral analysis is carried out using 128-points blocks with edge tapering (Hanning window) and 50\% overlapping for each auto- and co-spectrum. In the end, an appropriate directional offset related to the local magnetic declination has been applied to the spectral estimates $a_{1}(f) \ldots b_{2}(f)$ (the buoys' North reference is actually magnetic: for the considered location and time period, it is about $6^{\circ}$ towards West). The recording details related to each buoy are listed in Table 1 below.

The fact the Waveriders' sampling frequency is thrice that of the Wavec buoy does not imply a better spectral estimation since it only modifies the Nyquist frequency, which is here 
higher than $0.5 \mathrm{~Hz}-$ a common and natural cut-off high frequency for the observation of gravity waves - for all buoys. On the other hand, the spectral estimation is affected by the recording duration - through the frequency resolution - which is shorter on the Wavec buoy (1200s instead of 1600s for the Waveriders). However, the further spectro-directional processing applied to the data and the physical consideration of wave system partitions in the frequency-direction plane will make this difference negligible.

Fig. 4

Table 1

\subsection{Spectral data processing}

All three directional re-composition methods $-\cos ^{2 \mathrm{~s}}$ parametric, Burg's and Shannon's MEM - have been applied in both datasets with a common fine directional resolution of $\Delta \theta=$ $10^{\circ}$. An illustration is given in Figure 5 where a multimodal sea state spectrum has been recomposed from OCEANOR spectral data (DW1, $5^{\text {th }}$ of March 1994). In either case, the directional spectrum has been smoothed thanks to a simple moving average $2 \mathrm{D}$-window as defined in [18], and possibly repeated a couple of times according to the noise and sharpness of the raw spectrum, based on empirical observations. Smoothing is important here for the partitioning algorithm in SPOP, which is much facilitated when inputting smoothed directional spectral densities. Thus, the $\cos ^{2 \mathrm{~s}}$ parametric re-composition is followed by 2-loop smoothing, Burg's MEM by 3-loop smoothing and Shannon's MEM by 1-loop smoothing. Burg's MEM was rapidly abandoned for our purpose, however, because of its sensitivity to directional resolution (narrow peaks) - requiring more smoothing - and the possible presence of numerical artefacts in spite of the smoothing as stressed previously in $\S 2.2 .3$. Accordingly, Shannon's MEM was kept as the default implemented method and the $\cos ^{2 \mathrm{~s}}$ parametric 
method was used as an auxiliary one, as explained in the following. For each spectrum and both methods, it was checked that the adopted directional resolution and the smoothing were not significantly modifying overall wave parameters like $H_{\mathrm{m} 0}$ with respect to the raw values.

Fig. 5

While processing the PHI buoy data (DW3\&DW4) an unexpected phenomenon has been noticed in the computed spectra, which was not visible in the OCEANOR data. For both ME methods, a secondary "reflected" peak - whose energy is not negligible (20-30\%) - was often noticed around the same peak frequency as the main system (Fig. 6). To the knowledge of the authors, such a particularity has not been explicitly reported so far in any scientific publication about the directional re-composition unfortunately. The phenomenon could not be due to wave reflection at the shoreline as only infra-gravity waves could theoretically be reflected up to such a long distance from the coast. It was finally admitted that the phenomenon was inherent to the ME methods applied to the PHI directional wave spectral data. The resulting mean directions and mean spreading calculated in PHI spectra are correct, but the directional distributions - wrongly bimodal here - are very much likely to be erroneous compared with OCEANOR ones. Indeed, the secondary system observed in Figure $6 \mathrm{f} \& \mathrm{~h}\left(120-130^{\circ}\right)$ corresponds to that obtained with the truncated Fourier series (Fig. 6b, also observed in Fig. 6a). The MEM applied to PHI data seems not to be able to include this energy into the main swell peak $\left(\sim 315^{\circ}\right)$ as in the case of OCEANOR data (Fig. 6e\&g). As a consequence, it was decided to apply the $\cos ^{2 \mathrm{~s}}$ parametric method instead of Shannon's MEM for PHI data, since the sea states were not exhibiting more than one peak at each frequency in a large majority according to the corresponding OCEANOR spectra. The directional distributions were then estimated using equations (10) and (11) and each spectrum was smoothed only once. 
Fig. 6

\section{Sea state statistics}

\subsection{Unimodal characterisation}

The following 2-entry diagrams against the overall wave parameters given in equation (24) are plotted in Figure 7 for buoys DW1, DW2 and DW3 respectively, as: $H_{\mathrm{m} 0}-T_{-10}, H_{\mathrm{m} 0}$ $-T_{\mathrm{p}}, H_{\mathrm{m} 0}-\theta_{\mathrm{m}}, P_{\mathrm{w}}-\theta_{\mathrm{m}}, \Lambda-T_{-10}$, and $\sigma_{\mathrm{m}}-T_{\mathrm{p}}$.

$H_{\mathrm{m} 0}-T_{-10}$ and $H_{\mathrm{m} 0}-T_{\mathrm{p}}$ diagrams are quite common in wave energy, but it is of interest to figure out the noticeable differences they reveal. $T_{\mathrm{p}}$ tracks the main spectral peak, which correspond to swell systems most of the time: this accounts for the high period values reached in the diagrams (11-13s). No information is provided about secondary systems though. In turn, $H_{\mathrm{m} 0}-T_{-10}$ diagrams depict two particular sea state regimes dominating the considered weather window: the one around $7.5 \mathrm{~s}$ and the second around $10 \mathrm{~s}$, which correspond to mixed and swell-dominated sea states respectively. Directional diagrams $H_{\mathrm{m} 0}-$ $\theta_{\mathrm{m}}$ and $P_{\mathrm{w}}-\theta_{\mathrm{m}}$ show similar results: the main wave energy arises from North-West (angular sector centred on $315^{\circ}$ ) whatever the location, with $H_{\mathrm{m} 0} \sim 2.5 \mathrm{~m}$ and $P_{\mathrm{w}} \sim 30 \mathrm{~kW} . \mathrm{m}^{-1}$ offshore (DW1, DW3) and $H_{\mathrm{m} 0} \sim 2 \mathrm{~m}$ and $P_{\mathrm{w}} \sim 15 \mathrm{~kW} \cdot \mathrm{m}^{-1}$ nearshore (DW2). $\Lambda-T_{-10}$ diagrams are quite interesting for they clearly illustrate the fact the bandwidth increases as the mean wave period $T_{-10}$ decreases. A very similar oyster-shaped distribution is obtained at each buoy location. This information is of the highest interest for the design of oscillating offshore structures such as WECs since it helps tuning up their response to wave excitation - resonance peak and sensitivity width - according to the local field statistics, see [3]. Finally, $\sigma_{\mathrm{m}}-T_{\mathrm{p}}$ diagrams do 
not seem very informative since $\sigma_{\mathrm{m}}$ does not vary a lot against $T_{\mathrm{p}}$, as well as it appears highly dependent on the buoy type and the directional re-composition $\left(\sigma_{\mathrm{m}} \sim 25-30^{\circ}\right.$ for DW1 and DW2, $\sigma_{\mathrm{m}} \sim 40-45^{\circ}$ for DW3).

Also noticeable that diagrams are very close to each other whatever the location and data source - except for the case of directional spreading and wave energy/power $\left(H_{\mathrm{m} 0}\right.$ and $\left.P_{\mathrm{w}}\right)$, which is slightly absorbed as waves arrive to the shore. A clear statistical agreement is therefore obtained between buoys DW1, DW3, and - to a lesser extent DW2 -, firstly, because of the quality of the buoy data, and secondly, because of the widely homogeneous wave conditions observed in the oceanic region of Figueira da Foz over the considered period of time.

Fig. 7

\subsection{Multi-system characterisation}

The spectra are processed with SPOP according to $\S 3.2$ so that statistics can be drawn for each kind of wave system. Diagrams are built from the computation of the system parameters in equation (29), as: $H_{\mathrm{m} 0}-T_{\mathrm{p}}, H_{\mathrm{m} 0}-\theta_{\mathrm{p}}, P_{\mathrm{wa}}-\theta_{\mathrm{p}}, \lambda-T_{\mathrm{p}}$, and $\sigma-T_{\mathrm{p}}$. Swell-related diagrams are plotted in Figure 8 for buoys DW1, DW2 and DW3; wind-sea-related ones are shown in Figure 9.

Each type of diagram - swell or wind-sea - emphasizes the non-negligible discrepancies existing between both systems in terms of wave characteristics in Figueira da Foz (offshore and nearshore). Firstly, $H_{\mathrm{m} 0}-T_{\mathrm{p}}$ diagrams underline the different most frequent energy levels reached by both (peak values), as: for swells, $H_{\mathrm{m} 0} \sim 2 \mathrm{~m}$ offshore and $H_{\mathrm{m} 0} \sim$ $1.75 \mathrm{~m}$ nearshore, while $T_{\mathrm{p}} \sim 12 \mathrm{~s}$ whatever the distance to shore; for wind-seas, $H_{\mathrm{m} 0} \sim 1 \mathrm{~m}$ offshore and nearshore while $T_{\mathrm{p}} \sim 5 \mathrm{~s}$. This was expected because the nearshore energy 
dissipation of short waves is theoretically much lower than for long ones for they are less influenced by the sea bottom. For both kinds of systems, it is observed that the peak period of the system is not influenced by the bathymetry either, which was expected too. Also, directional diagrams $H_{\mathrm{m} 0}-\theta_{\mathrm{p}}$ clearly highlight that swells arise within a restricted NorthWestern angular sector $\left(\sim 315^{\circ}\right)$ - except nearshore where refraction deflects the wave crests according to bathymetry $\left(\sim 285^{\circ}\right)$; wind-seas also propagate within a restricted sector centred on North-West-North $\left(\sim 345^{\circ}\right)$ - except nearshore where this sector is much broader and centred on $\sim 330^{\circ}$. Swell spectral bandwidths never exceed $0.07-0.09 \mathrm{~Hz}$, while for wind-seas $\lambda$ may sometimes reach $0.25 \mathrm{~Hz}$ : the same oyster pattern as in the overall characterisation case (Fig. 7) is observed. Power levels never exceed $15 \mathrm{~kW} \cdot \mathrm{m}^{-1}$ for wind-seas, especially offshore (extremely weak power nearshore); wave power levels for swells are rather superior to this value and range from $10 \mathrm{~kW} \cdot \mathrm{m}^{-1}$ to $45 \mathrm{~kW} \cdot \mathrm{m}^{-1}$. Finally, the diagrams related to directional spreading, once again, do not permit to detect any particular trend for each type of system: all $\sigma$ values mostly keep close to each other against $T_{\mathrm{p}}$ according to the buoy type (around $32.5^{\circ}$ for OCEANOR's DW1 and DW2) except for PHI's DW3, which exhibits distinct peak spreading values for swells and wind-seas, as around $37.5^{\circ}$ and $42.5^{\circ}$ respectively. It yet remains that the (mean) directional spreading is a highly sensitive characteristic to deal with for wave systems as well as for the whole sea state spectrum.

Let us underline that the automatic classification of wave systems into swell and windsea operated by SPOP is subject to some user-defined physical criteria. This is why some systems may be identified as swells instead of wind-seas and vice versa, which may have originated some distortion in the final system statistics. However, the resulting system diagrams obtained from various data sources (buoy types and water depths) permit to satisfactorily separate the specific properties of swell and wind-sea systems. Each type has its own advantages in view of harnessing wave energy, as observed in that location during the March to May 1994 period. Swell characteristics do not significantly vary here, especially 
peak direction and spectral bandwidth, so that variations of the low-frequency response of slack- or tight-moored floating structures also should remain small. On the contrary, windseas are more variable systems, which, on the one hand, can be an issue for the orientation of the WECs if these are not axi-symmetrical for instance, but be positive on the other hand for the spectral bandwidths are much broader, which may ensure more homogeneous operating conditions. Let us repeat that an oscillating WEC - in particular a small single unit - deployed in the Atlantic Ocean will mostly be excited by wind-seas because of its limited physical dimensions, which generally does not allow for sensitivity below $0.10-12 \mathrm{~Hz}$. Also, part of the low-frequency motions originated by swell waves might be undesirable and have to be taken into account in the design of mooring systems. Similarly, as already said above, wind-seas might somewhat influence the heading of the structure in case the yawing motions are not restrained.

Figure 10 depicts histograms related to the sea state multimodality observed at each buoy location and classified according to the wave contents as follows: one swell only ("1SW"), one wind-sea only ("1WS"), mixed sea with one swell and one wind-sea (“1SW+1WS"), mixed sea with two swells (“2SW"), mixed sea with two swells and one wind-sea ("2SW+1WS"), mixed sea with two wind-seas ("2WS") and others (more systems). A very good agreement is found between the two OCEANOR buoys DW1 and DW2 ( 65\% "1SW", $12 \%$ "1WS", $18 \%$ "1SW+1WS" and $~ 3 \%$ "2SW"), which confirms that no physical change is observed as the systems propagate from the open ocean to the shore. The statistics obtained with PHI DW3 buoy data are slightly different however, especially the classification of mixed seas ( $262 \%$ "1SW", $\sim 10 \%$ "WS", $\sim 26 \%$ " $1 \mathrm{SW}+1 \mathrm{WS}$ " and $\sim 1 \%$ " $2 \mathrm{SW}$ "), but remain consistent with the overall wave climate experienced at that location. It seems, therefore, that the classification algorithm is more sensitive to the type of data source (buoy) - including the directional re-composition - than the bathymetry in the present case. Accordingly, it is recommended to use a homogenous in situ measurement network when 
investigating the wave contents and the directional properties of sea states, so that such characteristics can be compared on the same basis. The multimodality statistics also permit to look back into the unimodal description and justify its use in the present study, for unimodal sea states occurred $70-80 \%$ of time in the considered period.

Fig.8

Fig. 9

Fig. 10

\section{Conclusions}

This study has involved measured directional data coming from three independent buoys contemporaneously deployed in the Portuguese area of Figueira da Foz at several depths (20, 72 and 92m). Two buoys were directional Waveriders (OCEANOR) while the third one was a directional Wavec device (PHI). According to the type of raw data, a specific data processing has been applied for each. Wave spectra estimated from the Waverider buoys data were recomposed by maximum entropy (ME) method based on Shannon's formulation, whilst those estimated by the Wavec buoy were recomposed using a simple $\cos ^{2 \mathrm{~s}}$ parametric method due to non-physical artefacts appearing in the spectra when applying ME methods. The total recording duration under consideration in this study spanned 3 months (March to May 1994).

The unimodal characterisation - physically acceptable in that location - of the sea states recorded in that period included parameters accounting for energy $\left(H_{\mathrm{m} 0}\right)$, period $\left(T_{\mathrm{p}}, T_{\text {- }}\right.$ 10), wave power $\left(P_{\mathrm{w}}\right)$, direction $\left(\theta_{\mathrm{m}}\right)$, spectral bandwidth $(\Lambda)$ and directional spreading $\left(\sigma_{\mathrm{m}}\right)$. The multi-system approach permitted to characterise separately each wave system making up 
the sea state in a very similar way $\left(\left\{H_{\mathrm{m} 0}, T_{\mathrm{p}}, \theta_{\mathrm{p}}, P_{\mathrm{wa}}, \lambda, \sigma\right\}\right.$ for each system). Both approaches yielded long-term wave climate statistics as 2-entry occurrence diagrams involving these parameters. The multi-system analysis also permitted to derive statistics related to the multimodality of sea states, as swell alone, mixed sea with one swell and one wind-sea, etc. This case study - although carried out over quite a short period of time - has led to the following conclusions and recommendations for future similar investigations.

The directional spreading parameter $\left(\sigma_{\mathrm{m}}, \sigma\right)$ showed to be weakly relevant in both characterisations. Indeed, it has shown to depend a lot on the directional processing (resolution, re-composition method, smoothing) without exhibiting any clear correlation to wave period. On the contrary, the spectral bandwidth of the sea state - in both the unimodal ( $\Lambda$ ) and multi-system $(\lambda)$ characterisations - has proved itself as a key new characteristic for resource assessment purposes. A clear correlation to (mean and peak) wave period has been observed, which may help WEC developers design their devices - in particular the sensitivity bandwidth around the resonance frequency - according to the own bandwidth of the wave field. In addition, it was seen that swell and wind-sea systems had very distinct bandwidth ranges.

This study has also emphasized the homogeneity of the wave conditions near Figueira da Foz - offshore and nearshore - during the considered period of time. Very similar statistics have been obtained from OCEANOR and PHI data indeed (out of obvious refraction and energy dissipation effects), except directional spreading which was too much data-sourcedependent and sensitive to re-composition, as already stressed above. For such investigation purposes, it is recommended that the measurement network be composed of identical devices, so that the data linked to wave directionality spreading may be compared on the same basis as soon as the same spectro-directional processing is applied to the raw buoy data.

Lastly, the partitioning and classification algorithm implemented in SPOP has shown to provide consistent statistical results on sea state contents from two different data sources 
related to close locations (DW1\&DW3), although they might- once again - be sensitive to the data source (buoy type) and the directional re-composition method, which may eventually affect the determination of the sea state type (mixed seas in particular). Very similar sea state multimodality histograms were found with OCEANOR data offshore and nearshore however, so that the wave system partitioning and classification procedures seemed consistent with ocean physics.

\section{Acknowledgements}

This work was funded by the E. C. EQUIMAR project (grant agreement FP7-213380). The authors thank the Portuguese Hydrographic Institute (Instituto Hidrográfico) for the free use of Wavec buoy data in Figueira da Foz. They also thank H. E. Krogstad for his personal recommendations concerning the numerical implementation of ME methods.

\section{References}

[1] R. Waters, J. Engström, J. Isberg, M. Leijon, Wave climate off the Swedish west coast, Renewable Energy 34 (2009) 1600-1606.

[2] P. Lenee-Bluhm, R. Paash, H.T. Özkan-Haller, Characterizing the wave energy resource of the US Pacific Northwest, Renewable Energy 36 (2011) 2106-2119.

[3] J.-B. Saulnier, A. Clément, A.F. de O. Falcão, T. Pontes, M. Prevosto, P. Ricci, Wave groupiness and spectral bandwidth as relevant parameters for the performance assessment of wave energy converters, Ocean Eng. 38 (2011) 130-147. 
[4] H. Mitsuyasu, F. Tasai, T. Suhara, S. Mizuno, M. Ohkusu, T. Honda, K. Rikiishi, K., Observations of the directional spectrum of ocean waves using a cloverleaf buoy, J. Phys. Oceanogr. 5 (1975) 750-760.

[5] L.E. Borgman, Estimation of wave directional spectra from pitch-roll buoy data, J.

Waterways, Port., Coastal and Ocean Eng. 120 (1969) 93-115.

[6] R.B. Long, The statistical evaluation of directional spectrum estimates derived from pitch/roll buoy data, J. Phys. Oceanogr. 10 (1980) 944-952.

[7] M. Benoit, P. Frigaard, A. Schäffer, Analysing multidirectional wave spectra: a tentative classification of available methods, in: Proc. IAHR'97, Seminar on Multidirectional waves and their interaction with structures, San Francisco, 1997, pp. 131-158.

[8] H.E. Krogstad, S.F. Barstow, O. Haug, D.J.H. Peters, Directional distributions in wave spectra, in: Proc. Ocean Wave Measmts. and Anal. Conf., 2004, pp. 883-895.

[9] J.P. Burg, Maximum entropy spectral analysis, PhD Thesis, Stanford University, 1975.

[10] K. Kobune, N. Hashimoto, Estimation of directional spectra from the maximum entropy principle, in: Proc. $5^{\text {th }}$ Int. Offshore Mech. and Arct. Eng. Symp., Tokyo, 1986, pp. 80-85. [11] O.U. Nwogu, E.P.D. Mansard, M.D. Miles, M. Isaacson, Estimation of directional wave spectra by the maximum entropy method, in: Proc. $17^{\text {th }}$ IAHR Seminar on Wave generation and analysis in laboratory wave basins, Lausanne, 1987.

[12] A. Lygre, H.E. Krogstad, Maximum entropy estimation of the directional distribution in ocean wave spectra, J. Phys. Oceanogr. 16 (1986) 2052-2060.

[13] M.D. Earle, K.E. Steele, D.W.C. Wand, Use of advanced directional wave spectra analysis methods, Ocean Eng. (Technical note) 26 (1999) 1421-1434.

[14] R.B. Blackman, J.M. Tukey, The measurement of power spectra, Dover Publications Inc., New-York, 1959. 
[15] M.-A. Kerbiriou, M. Prevosto, C. Maisondieu, Influence of an improved sea-state description on a wave energy converter production, in: Proc. $26^{\text {th }}$ Int. Conf. on Offshore Mech. and Arct. Eng., San Diego, 2007, pp. 463-473.

[16] S. Hasselmann, C. Brüning, K. Hasselmann, P. Heimbach, An improved algorithm for retrieval of ocean wave spectra from synthetic aperture radar image spectra, J. Geophys. Res. 101 (1996) 16615-16629.

[17] J.L. Hanson, O.M. Phillips, Automated analysis of ocean surface directional wave spectra, J. Atmos. and Ocean Tech. 18 (2001) 277-293.

[18] J. Portilla, F.J. Ocampo-Torres, J. Monbaliu, Spectral partitioning and identification of wind sea and swell, J. of Atmos. and Ocean Tech. 26 (2009) 107-122. 


\begin{tabular}{lccc}
\hline Characteristics & $\boldsymbol{D} \boldsymbol{W} \mathbf{1}$ & $\boldsymbol{D} \boldsymbol{W} \mathbf{2}$ & $\boldsymbol{D W}$ \\
\hline Data provider & OCEANOR & OCEANOR & PHI \\
Buoy type & Waverider dir. & Waverider dir. & Wavec dir. \\
Recorded data & Heave/North/West disp. & Heave/North/West disp. & Heave/Pitch/Roll \\
Location & $40^{\circ} 15^{\prime} 04^{\prime \prime N}$, & $40^{\circ} 12^{\prime} 24^{\prime \prime} \mathrm{N}$, & $40^{\circ} 11^{\prime} 08^{\prime}{ }^{\prime}$, \\
& $09^{\circ} 04^{\prime} 59^{\prime \prime} \mathrm{W}$ & $08^{\circ} 55^{\prime} 55^{\prime} \mathrm{W}$ & $09^{\circ} 08^{\prime} 44^{\prime \prime} \mathrm{W}$ \\
Mean water depth & $\sim 72 \mathrm{~m}$ & $\sim 20 \mathrm{~m}$ & $\sim 92 \mathrm{~m}$ \\
Operating duration & $1994 / 03 / 04$ & $1994 / 03 / 04$ & $1994 / 03 / 01$ \\
& $1994 / 05 / 26$ & $1994 / 05 / 26$ & $1994 / 06 / 24$ \\
Sampling frequency & $3.84 \mathrm{~Hz}$ & $3.84 \mathrm{~Hz}$ & $1.28 \mathrm{~Hz}$ \\
Recording duration & $1600 \mathrm{~s}$ & $1600 \mathrm{~s}$ & $1200 \mathrm{~s}$ \\
Recording intervals & $3 \mathrm{~h}$ & $3 \mathrm{~h}$ & $3 \mathrm{~h}$ \\
\hline
\end{tabular}

Table 1: Characteristics of buoys OCEANOR and PHI buoys deployed near Figueira da Foz (Portugal). 


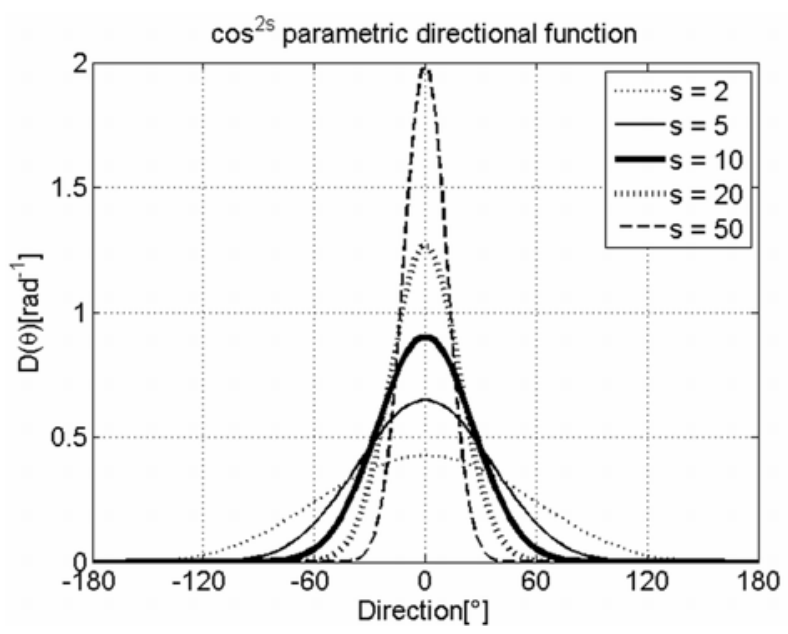

Fig. 1: $\operatorname{Cos}^{2 \mathrm{~s}}$ parametric directional function for various spreading values (s). 


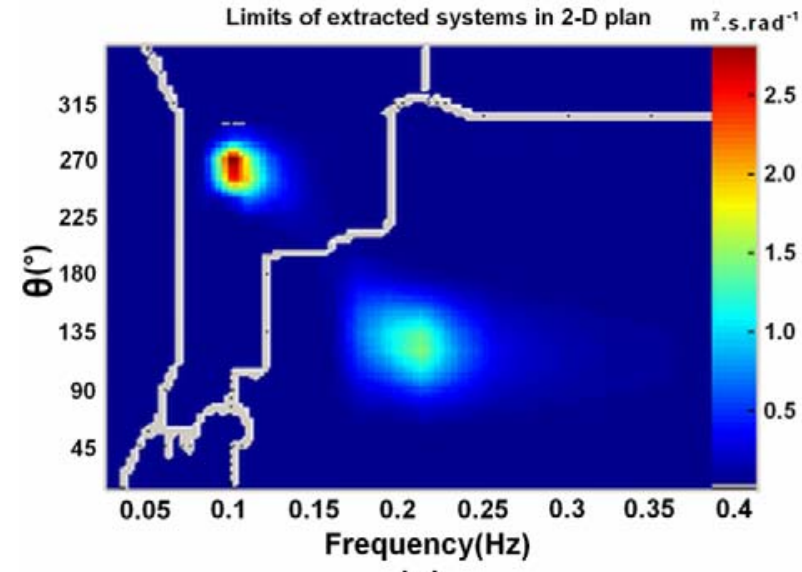

(a)

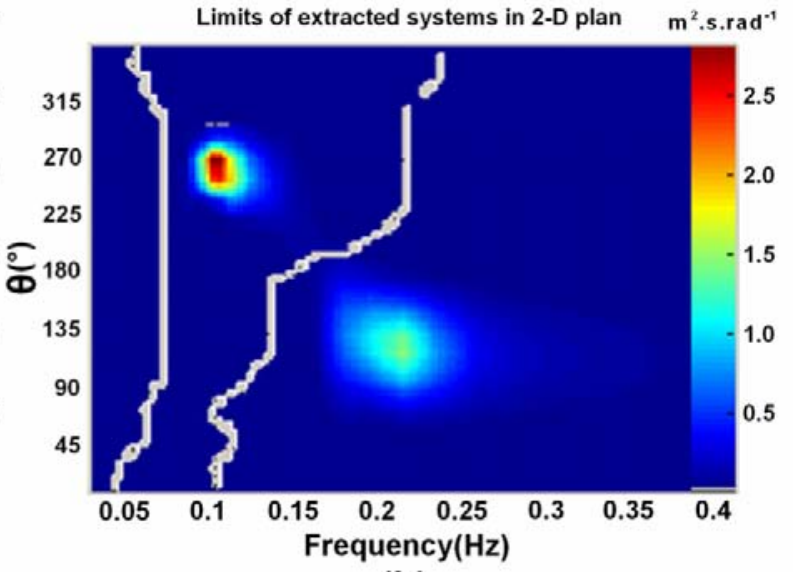

(b)

Fig. 2: Wave spectrum partitioning before (a) and after (b) wave system grouping (SPOP). 


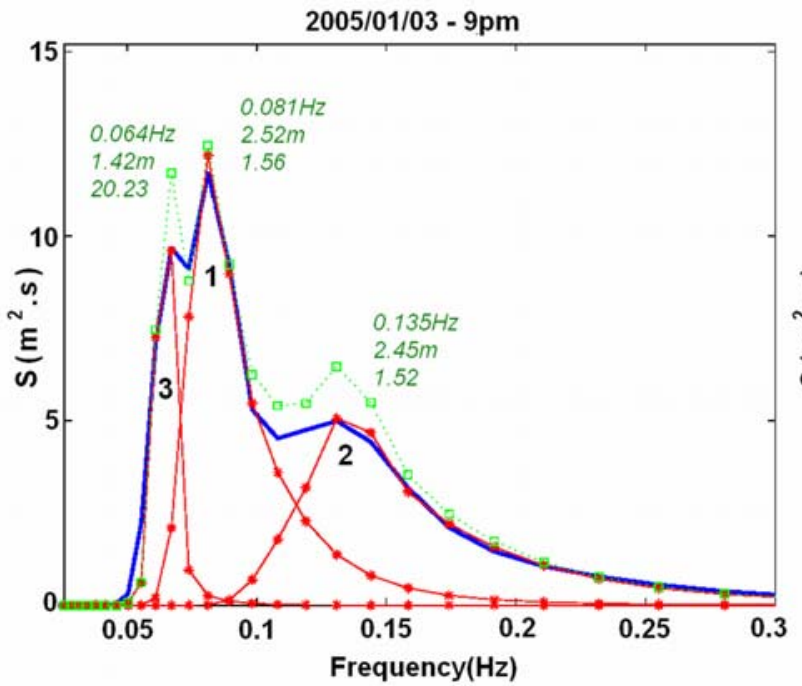

(a)

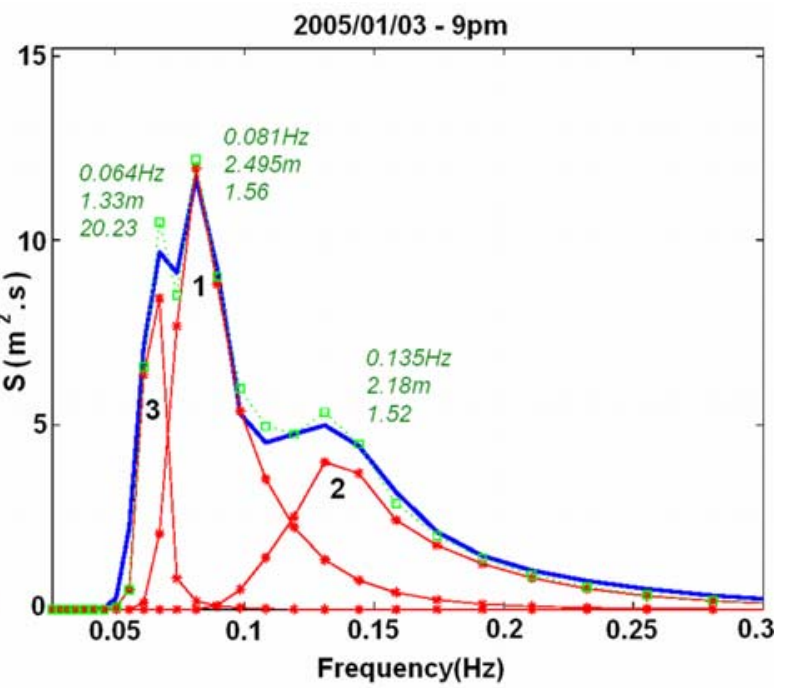

(b)

Fig. 3 : Wave system fitting with parametric spectral shapes (modified JONSWAP) without (a) and with (b) mutual influence correction. 


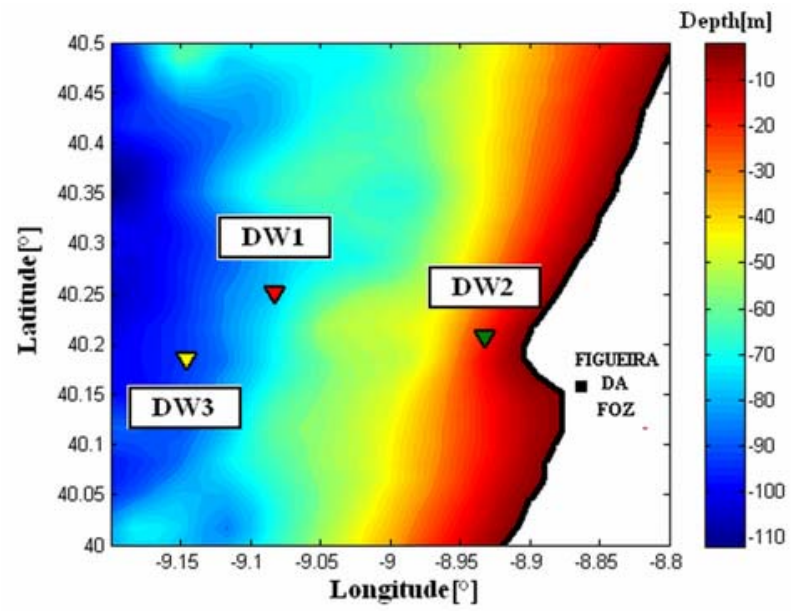

Fig. 4 : Bathymetry and location of buoys DW1 (72m), DW2 (20m) and DW3 (92m) near Figueira da Foz (Portugal). 

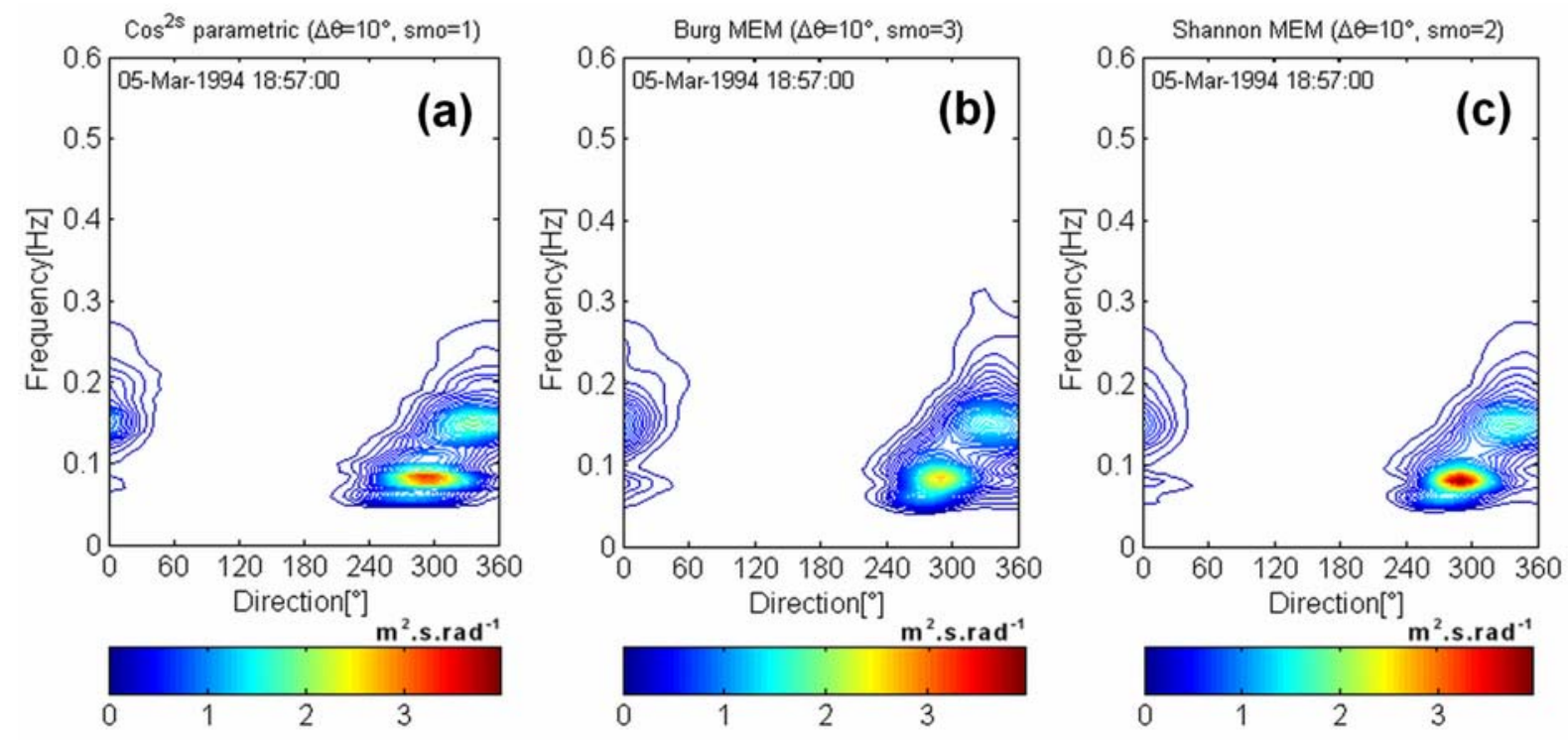

Fig. 5 : Directional wave spectrum estimated using parametric method with $\cos ^{2 \mathrm{~s}}$ function (a) and maximum entropy method with Burg's (b) and Shannon's (c) formulation. 

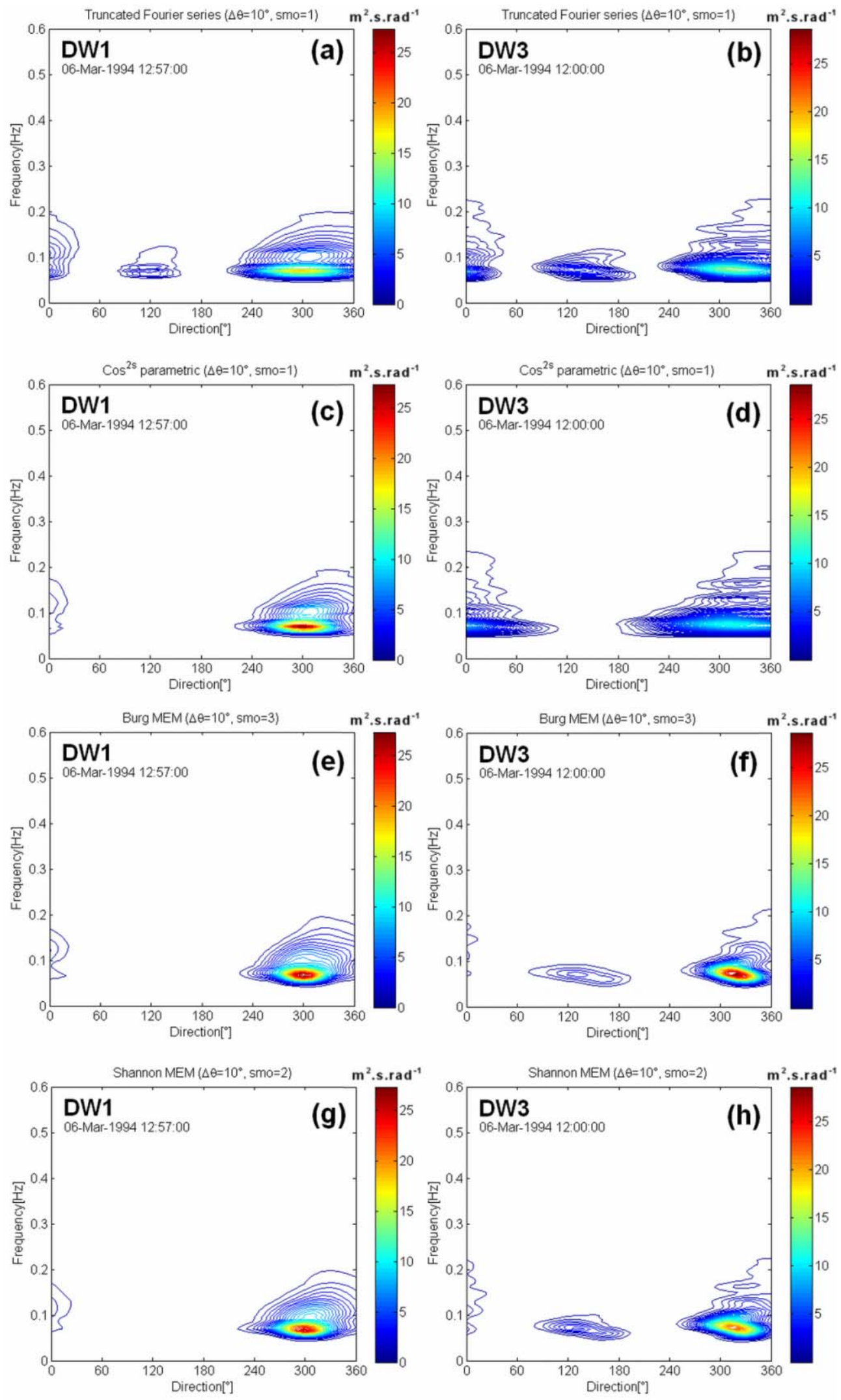

Fig. 6: Directional recomposition of the wave spectrum at DW1 (left) and DW3 (right) buoy locations on the $6^{\text {th }}$ of March 1994, 12am-1pm: truncated Fourier series (a\&b), cos2s function (c\&d), Burg MEM (e\&f), Shannon MEM (g\&h). 

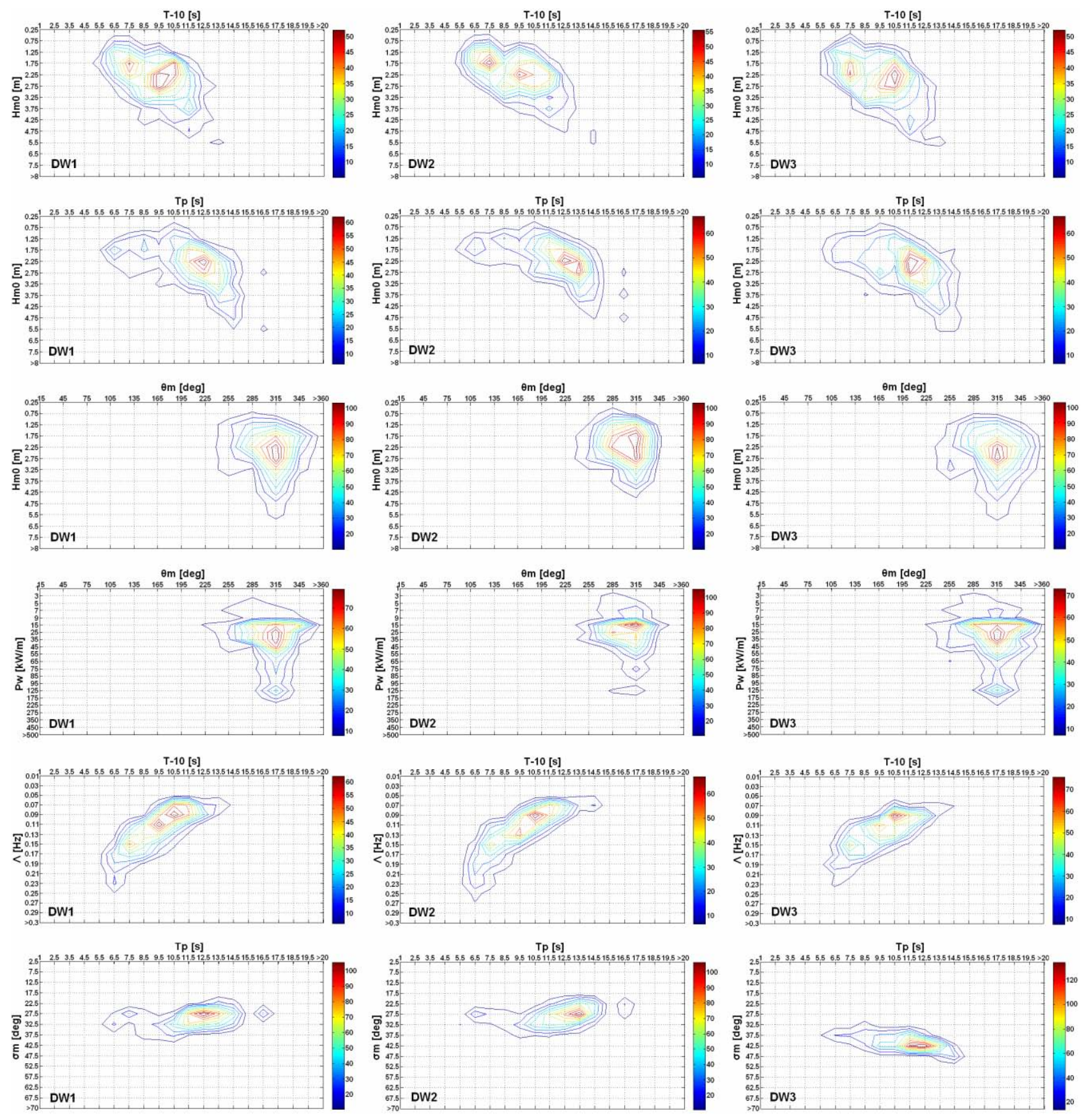

Fig. 7 : Scatter diagrams of overall parameters at DW1 (left), DW2 (centre) and DW3 (right) buoy locations (from top to bottom) : $H_{\mathrm{m} 0}-T_{-10}, H_{\mathrm{m} 0}-T_{\mathrm{p}}, H_{\mathrm{m} 0}-\theta_{\mathrm{m}}, P_{\mathrm{w}}-\theta_{\mathrm{m}}, \Lambda-T_{-10}$ and $\sigma_{\mathrm{m}}-T_{\mathrm{p}}$ (Figueira da Foz, March-May 1994) 

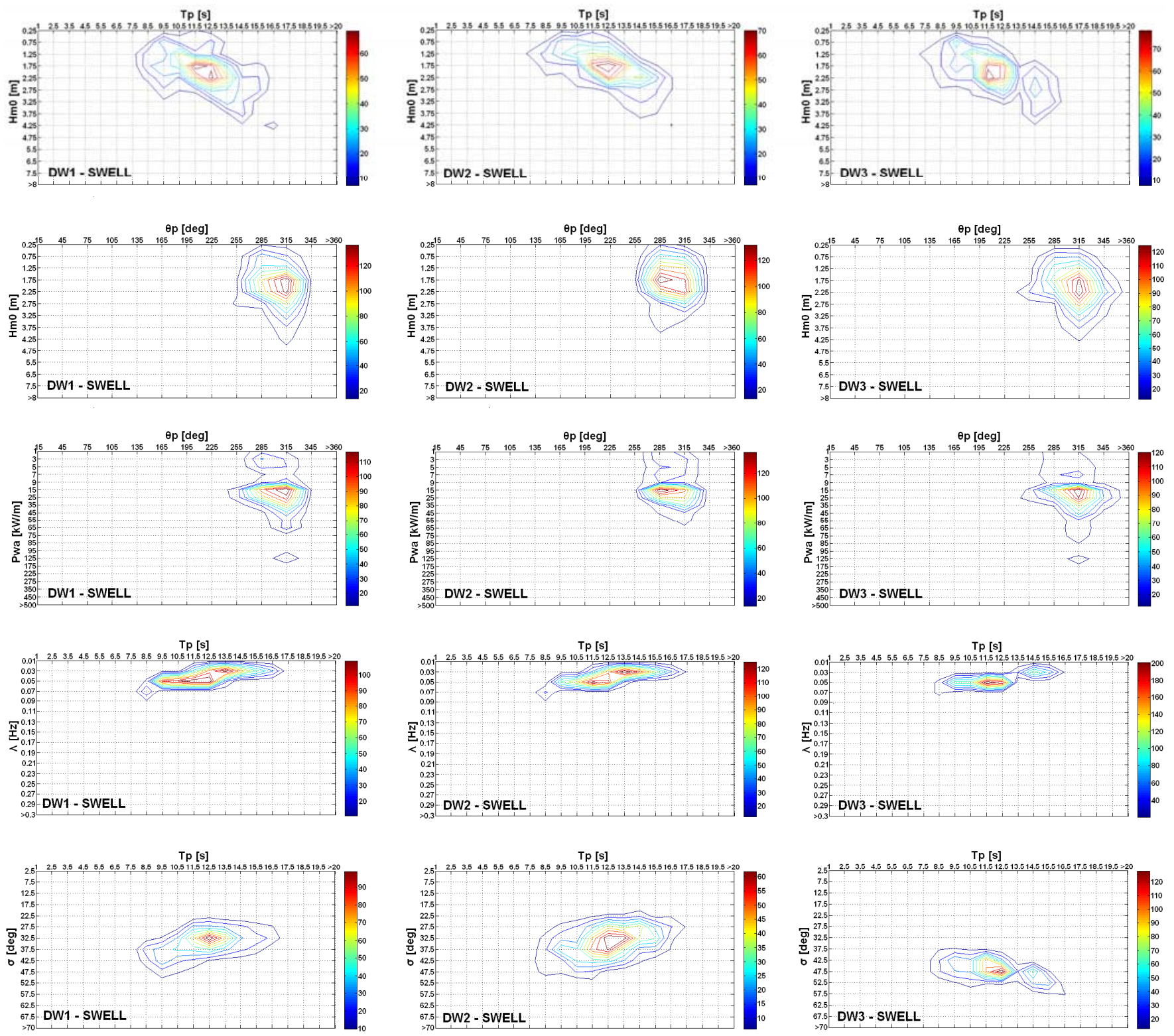

Fig. 8 : Scatter diagrams of swell parameters at DW1 (left), DW2 (centre) and DW3 (right) buoy locations (from top to bottom): $H_{\mathrm{m} 0}-T_{\mathrm{p}}, H_{\mathrm{m} 0}-\theta_{\mathrm{p}}, P_{\mathrm{wa}}-\theta_{\mathrm{p}}, \lambda-T_{\mathrm{p}}$ and $\sigma-T_{\mathrm{p}}$ (Figueira da Foz, March-May 1994). 

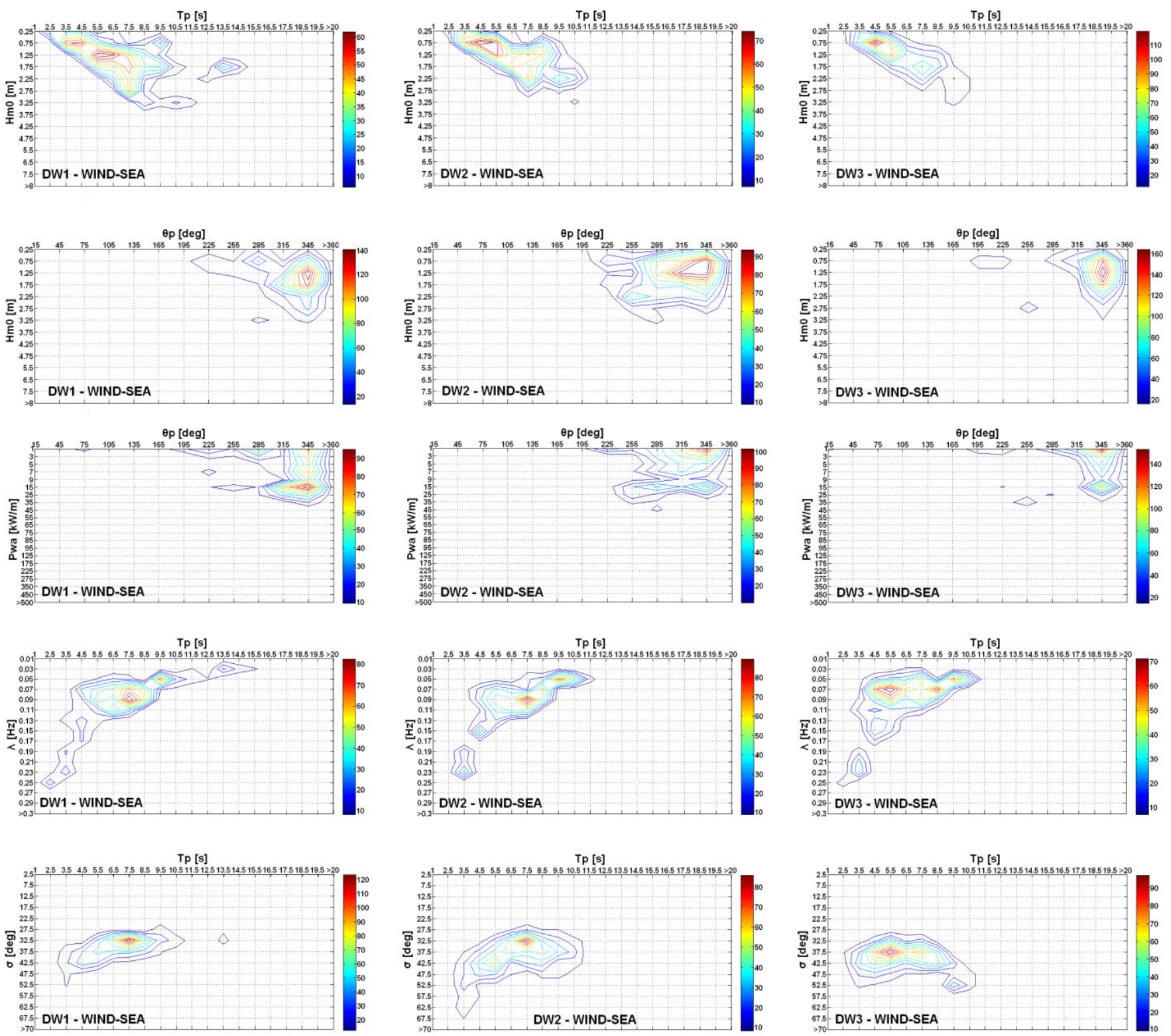

Fig. 9 : Scatter diagrams of wind-sea parameters at DW1 (left), DW2 (centre) and DW3 (right) buoy locations (from top to bottom): $H_{\mathrm{m} 0}-T_{\mathrm{p}}, H_{\mathrm{m} 0}-\theta_{\mathrm{p}}, P_{\mathrm{wa}}-\theta_{\mathrm{p}}, \lambda-T_{\mathrm{p}}$ and $\sigma-T_{\mathrm{p}}$ (Figueira da Foz, March-May 1994). 

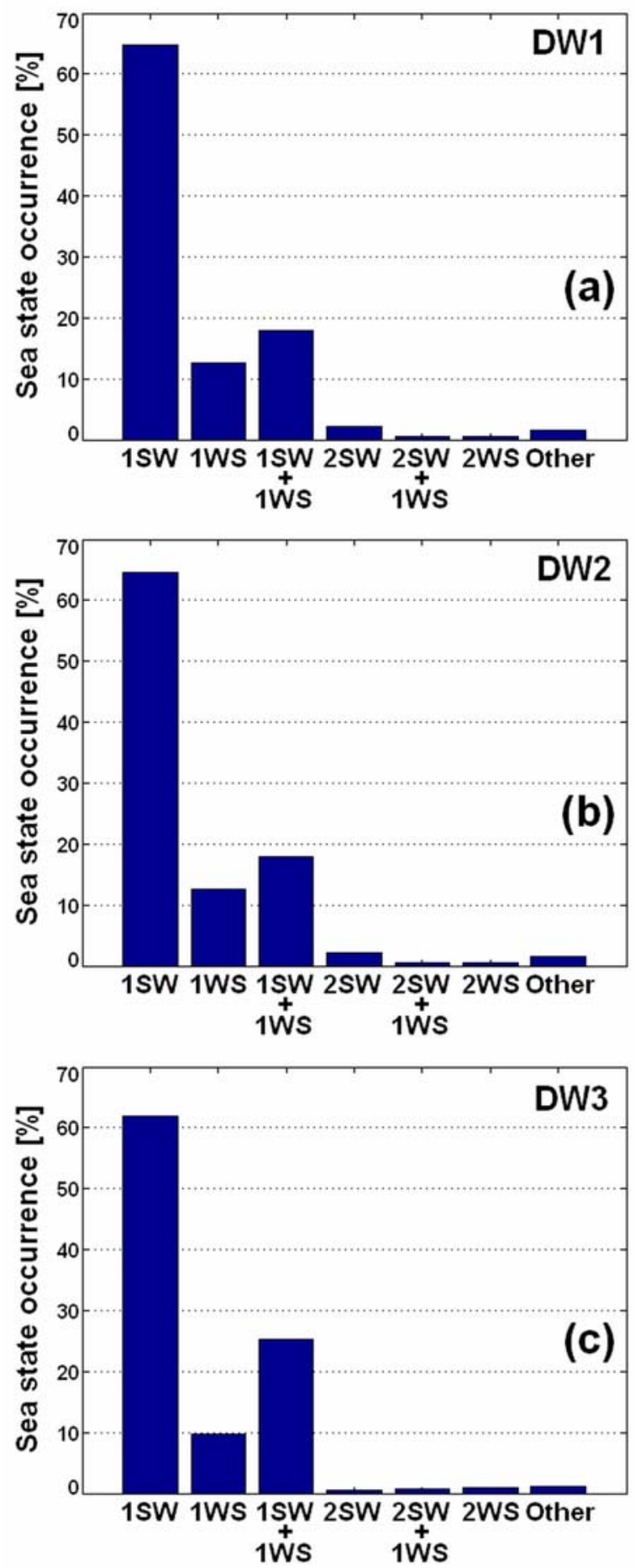

Fig. 10 : Sea state occurrences (\%) at DW1 (a), DW2 (b) and DW3 (c) buoy locations according to the type (swell- or wind-sea-dominated and mixed seas). 